\title{
A MULTIPLYING FACTOR METHOD FOR THE SOLUTION OF WIENER-HOPF EQUATIONS $\dagger$
}

\author{
by B. NOBLE and A. S. PETERS
}

(Received 6th October 1960)

In (1), § 6.2, a multiplying factor method has been used to solve certain dual integral equations. The results are then used to solve a single integral equation of the Wiener-Hopf type. In this note we indicate how a related technique can be used to solve Wiener-Hopf integral equations directly. Consider

where

$$
\int_{0}^{\infty} k(x-\xi) f(\xi) d \xi=g(x), \quad(0<x<\infty),
$$

Define

$$
k(x)=O\left(e^{-p|x|}\right), \quad f(x)=O\left(e^{q x}\right), \quad-p<q<p .
$$

$$
F_{+}(\alpha)=\frac{1}{(2 \pi)^{1 / 2}} \int_{0}^{\infty} f(x) e^{i a x} d x, K(\alpha)=\frac{1}{(2 \pi)^{1 / 2}} \int_{-\infty}^{\infty} k(x) e^{i a x} d x,
$$

where $\alpha=\sigma+i \tau$, and $F_{+}(\alpha)$ is regular for $\tau>q ; K(\alpha)$ is regular and non-zero in $-p<\tau<p$. For simplicity we restrict ourselves to the case where

$$
|K(\alpha)| \sim|\alpha|^{-1} \text { as }|\alpha| \rightarrow \infty \text { in the strip }-p<\tau<p .
$$

We suppose also that $K(\alpha)$ can be decomposed in the form

$$
K(\alpha)=K_{+}(\alpha) K_{-}(\alpha),
$$

where $K_{+}(\alpha), K_{-}(\alpha)$ are regular and non-zero in $\tau>-p, \tau<p$ respectively, and $\left|K_{+}\right|,\left|K_{-}\right|<|\alpha|^{-1 / 2}$ as $\alpha \rightarrow \infty$ in their respective half-planes of regularity.

On applying the Fourier convolution theorem to the left-hand side of $(1 a)$,

$$
\int_{i a-\infty}^{i a+\infty} F_{+}(\alpha) K(\alpha) e^{-i \alpha x} d \alpha=g(x), \quad(x>0, q<a<p) .
$$

In this equation replace $x$ by $(x+\eta), x>0, \eta>0$; multiply by $\mathscr{L}(\eta)$ where this function is defined below, and integrate with respect to $\eta$ from 0 to $\infty$. Then

where

$$
\int_{i a-\infty}^{i a+\infty} F_{+}(\alpha) K(\alpha) L_{-}(\alpha) e^{-i \alpha x} d \alpha=\int_{0}^{\infty} \mathscr{L}(\eta) g(x+\eta) d \eta, \quad(x>0),
$$

$$
L_{-}(\alpha)=\int_{0}^{\infty} \mathscr{L}(\eta) e^{-i \alpha \eta} d \eta,
$$

$\dagger$ The research reported here has been sponsored by the Air Force Cambridge Research Center, Air Research and Development Command, under Contract No. AF 19(604)5238, and by the Office of Naval Research under Contract No. N-onr-285(46). 
i.e.

$$
\mathscr{L}(\eta)=\frac{1}{2 \pi} \int_{i b-\infty}^{i b+\infty} L_{-}(\alpha) e^{i \alpha \eta} d \alpha .
$$

From its definition $L_{-}(\alpha)$ is regular in some lower half-plane, say $\tau<p^{\prime}$; then we must choose $b<p^{\prime}$. From the assumptions on $K_{-}(\alpha)$ following (2) we can choose

$$
L_{-}(\alpha)=\left\{(\alpha-u) K_{-}(\alpha)\right\}^{-1},
$$

where $u$ is an arbitrary constant such that $\operatorname{Im} u \geqq p$. The factor $(\alpha-u)$ is introduced to ensure convergence of the integral in (4), where now $L_{-}(\alpha)$ is regular in $\tau<p$, and hence we must take $b<p$. The constant $u$ is at our disposal apart from the condition that it must lie in a specified upper half-plane. In the example given below there is a convenient and obvious choice for $u$.

Insertion of (5) in (2) gives

$$
\int_{i a-\infty}^{i a+\infty} F_{+}(\alpha) K_{+}(\alpha) \frac{e^{-i \alpha x} d \alpha}{\alpha-u}=\int_{0}^{\infty} \mathscr{L}(\eta) g(x+\eta) d \eta, \quad(x>0) .
$$

Multiply both sides by $\exp (i u x)$ and differentiate with respect to $x$. Then

$$
\begin{array}{r}
\int_{i a-\infty}^{i a+\infty} F_{+}(\alpha) K_{+}(\alpha) e^{-i \alpha x} d \alpha=i e^{-i u x} \frac{d}{d x} e^{i u x} \int_{0}^{\infty} \mathscr{L}(\eta) g(x+\eta) d \eta \\
=G(x), \text { say, }(x>0) . \ldots \ldots \ldots \ldots \ldots \ldots \ldots \ldots \ldots \ldots \ldots \ldots \ldots \ldots \ldots \ldots \ldots \ldots
\end{array}
$$

From the form of the integrand on the left-hand side the integral is automatically zero when $x<0$, so that the equation can be inverted to give

$$
\begin{aligned}
F_{+}(\alpha) & =\frac{1}{2 \pi K_{+}(\alpha)} \int_{0}^{\infty} G(y) e^{i \alpha y} d y, \\
f(x) & =\frac{1}{(2 \pi)^{3 / 2}} \int_{i c-\infty}^{i c+\infty} \frac{1}{K_{+}(\alpha)} e^{-i \alpha x} \int_{0}^{\infty} G(y) e^{i \alpha y} d y d a, \quad(q<c<p) .
\end{aligned}
$$

In order to allow interchange of orders of integration we write this as

$$
\begin{aligned}
& f(x)=\frac{i}{(2 \pi)^{3 / 2}} e^{-i v x} \frac{d}{d x} e^{i v x} \int_{i c-\infty}^{i c+\infty} \frac{e^{-i \alpha x}}{(\alpha-v) K_{+}(\alpha)} \int_{0}^{\infty} G(y) e^{i \alpha y} d y d a \\
& =\frac{i}{2 \pi} e^{-i v x} \frac{d}{d x} e^{i v x} \int_{0}^{x} \mathscr{M}(x-y) G(y) d y,
\end{aligned}
$$

where $v$ is a constant which can be chosen arbitrarily in a lower half-plane $\operatorname{Im} v \leqq-p$, and

$$
\mathscr{M}(\eta)=\frac{1}{(2 \pi)^{1 / 2}} \int_{i c-\infty}^{i c+\infty} \frac{e^{-i \alpha \eta}}{(\alpha-v) K_{+}(\alpha)} d a, \quad(\eta>0) .
$$


It follows that $\mathscr{M}(\eta)=0$ for $\eta<0$. On substituting for $G(y)$ in (7) from (6) we obtain the solution

$f(x)=-\frac{1}{2 \pi} e^{-i v x} \frac{d}{d x} e^{i v x} \int_{0}^{x} \mathscr{M}(x-y) e^{-i u y} \frac{d}{d y} e^{i u y} \int_{y}^{\infty} \mathscr{L}(\eta-y) g(\eta) d \eta d y$,

where $\operatorname{Im} u \geqq p, \operatorname{Im} v \leqq-p$.

As an application, consider

$$
\int_{0}^{\infty} K_{0}(\lambda|x-\xi|) f(\xi) d \xi=g(x), \quad(0<x<\infty),
$$

where $\operatorname{Re} \lambda>0$. Then $p=\operatorname{Re} \lambda$.

$$
K(\alpha)=(\pi / 2)^{1 / 2}\left(\alpha^{2}+\lambda^{2}\right)^{-1 / 2},
$$

and a suitable decomposition is given by

$$
K_{+}(\alpha)=(\pi / 2)^{1 / 2}(\alpha+i \lambda)^{-1 / 2}, \quad K_{-}(\alpha)=(\alpha-i \lambda)^{-1 / 2} .
$$

From (4), (5), (8), [cf. (1), ex. 2.4., p. 88], on choosing $u=-v=i \lambda$,

$$
\begin{aligned}
& \mathscr{L}(\eta)=\frac{1}{2 \pi} \int_{i b-\infty}^{i b+\infty}(\alpha-i \lambda)^{-1 / 2} e^{i \alpha \eta} d \alpha=\pi^{-1 / 2} e^{i \pi / 4} \eta^{-1 / 2} e^{-\lambda \eta}, \quad(\eta>0), \\
& \mathscr{M}(\eta)=\frac{1}{\pi} \int_{i c-\infty}^{i c+\infty}(\alpha+i \lambda)^{-1 / 2} e^{-i \alpha \eta} d \alpha=2 \pi^{-1 / 2} e^{-i \pi / 4} \eta^{-1 / 2} e^{-\lambda \eta}, \quad(\eta>0) .
\end{aligned}
$$

Hence (9) gives

$$
f(x)=-\frac{1}{\pi^{2}} e^{-\lambda x} \frac{d}{d x} \int_{0}^{x}(x-y)^{-1 / 2} e^{2 \lambda y} \frac{d}{d y} \int_{y}^{\infty}(\eta-y)^{-1 / 2} e^{-\lambda \eta} f(\eta) d \eta d y .
$$

This agrees with (1), (6.27), p. 228, on remembering that if we write

$$
\lambda=-i k, \operatorname{Im} k>0 \text {, then } K_{0}(\lambda x)=\frac{1}{2} i \pi H_{0}^{(1)}(k x) .
$$

A similar procedure can be used to solve

$$
\left(\frac{d}{d x}+i u\right)\left(\frac{d}{d x}+i v\right) \int_{0}^{\infty} k(x-\xi) f(\xi) d \xi=g(x), \quad(0<x<\infty),
$$

where we assume $\operatorname{Im} u=p_{1}>0, \operatorname{Im} v=-p_{2}<0$. Then instead of the condition on $q$ in $(1 b)$ we must have

$$
-\min \left(p, p_{1}, p_{2}\right)<q<\min \left(p, p_{1}, p_{2}\right) \text {. }
$$

In particular we can choose $u=-v=i \lambda$ so that the operator becomes $\left\{\left(d^{2} / d x^{2}\right)\right.$ $\left.-\lambda^{2}\right\}$. We make the same assumptions about $K(\alpha)$ as before. It is found that

$$
f(x)=-\frac{1}{2 \pi} \int_{0}^{x} \mathscr{M}(x-y) \int_{y}^{\infty} \mathscr{L}(\eta-y) g(\eta) d \eta d y,
$$

where the notation is exactly that defined previously and now no device is required to avoid divergent integrals. This solution is not unique unless further 
restrictions are imposed, since the form of (10) shows that we can add to (11) solutions of

$$
\int_{0}^{\infty} k(x-\xi) f(\xi) d \xi=A e^{-i u x}+B e^{-i v x},
$$

where $A, B$ are arbitrary constants. However (11) is the unique solution of (10) such that $f(x)$ tends to zero as $x$ tends to zero.

\section{REFERENCE}

(1) B. NoBLe, Methods based on the Wiener-Hopf technique for the solution of partial differential equations, Pergamon Press (1958).

The Royal College of Science and Technology, Glasgow

The Institute of Mathematical Sciences

New YoRK 